\title{
A potential scenario for Majorana neutrino detection at future lepton colliders
}

\section{Yang Zhang and Bin Zhang}

Department of Physics, Tsinghua University,

Beijing, 100084, China

Center for High Energy Physics, Tsinghua University,

Beijing, 100084, China

E-mail: zhang-yang13@mails.tsinghua.edu.cn, zb@mail.tsinghua.edu.cn

ABSTRACT: The existence of Majorana neutrinos must lead to lepton-number violating processes, and the Majorana nature of neutrinos can only be experimentally verified via lepton-number violating processes. We study an approach to search for Majorana neutrinos at future electron-positron colliders by exploiting this feature. We investigate $\Delta L=2$ likesign dilepton production and find that lepton colliders with different center-of-mass (c.m.) energies have comparative advantages in resonant production of a Majorana neutrino in both light and heavy neutrino mass range. At the future Circular Electron-Positron Collider (CEPC), with $250 \mathrm{GeV}$ c.m. energy and $5 \mathrm{ab}^{-1}$ integrated luminosity, we find that there could be more significant sensitivity for resonant production of a Majorana neutrino in the mass range of $30-50 \mathrm{GeV}$ than previous results at LEP2 or LHC. At the $1 \mathrm{TeV}$ ILC with 1 $\mathrm{ab}^{-1}$ integrated luminosity, the sensitivity can be improved for neutrino mass in the range of $450-600 \mathrm{GeV}$.

KeYwords: Beyond Standard Model, e+-e- Experiments

ARXiv EPRINT: 1805.09520 


\section{Contents}

1 Introduction 1

2 Models and mixing parameters $\quad 2$

3 Collider signatures $\quad \mathbf{5}$

3.1 Phenomenological Analysis at the CEPC 8

$\begin{array}{lll}3.2 & \text { Phenomenological analysis at the ILC } & 12\end{array}$

$\begin{array}{llr}4 & \text { Summary and conclusions } & 16\end{array}$

\section{Introduction}

A multitude of neutrino oscillation measurements [1, 2] from experiments with solar, atmospheric, reactor and accelerator neutrinos have shown that neutrinos have small non-zero masses, while the origin of their masses is still a mystery. The Standard model (SM), which incorporates left-handed neutrinos $\nu_{L}$ in $\mathrm{SU}(2)_{L}$ gauge group doublets, is unable to generate neutrino mass without giving up gauge symmetry and renormalizability, so additional particles must be added to extend the SM. The simplest scheme is to introduce $n$ right-handed SM singlet neutrino fields $N_{R}$. They can couple to the three flavour neutrinos through Yukawa couplings which result in Dirac mass terms $m_{D}\left(\overline{\nu_{L}} N_{R}+\right.$ h.c.) after gauge symmetry breaking. More interestingly, these gauge singlet neutrino fields are also allowed to couple to their own charge conjugate fields to form Majorana mass terms $M\left(\overline{N_{L}^{c}} N_{R}+\right.$ h.c. $)$. This is known as the type-I seesaw mechanism [3-8], which has strong theoretical motivation and can be incorporated into many scenarios, such as Left-Right symmetric gauge theories [9-11]; SO(10) Supersymmetric (SUSY) grand unification [1214] and other grand unified theories [15-18]; models with exotic Higgs representations [1924]; R-parity violating interactions in Supersymmetry (SUSY) [25] and theories with extra dimensions [26-28]. Introduction of Majorana masses which are absent in the SM and violate global U(1) symmetry, will result in lepton number no longer being conserved. Such unique phenomena, if observed, would provide the most unambiguous evidence for the existence of a Majorana neutrino. The observed neutrino flavour oscillation phenomena imply a mixture between the flavour and mass eigenstates of neutrinos. Therefore, via neutral current (NC) and charged current (CC) interactions, direct collider searches may produce lepton-number violating processes involving charged leptons which would help establish the nature of neutrino masses.

The prospects of detecting signals of heavy Majorana neutrinos at hadron colliders have been thoroughly explored [29-41]. Both the ATLAS and CMS experiments at the LHC have set limits on the mixing parameters for heavy Majorana neutrino masses between 1 
and $1600 \mathrm{GeV}$ [42-47]. The "smoking gun" signal at hadron colliders is Majorana neutrino production via the Drell-Yan (DY) process: $p p \rightarrow W^{*} \rightarrow N \ell^{ \pm} \rightarrow \ell^{ \pm} \ell^{ \pm} j j$. For Majorana neutrinos lighter than $W$ bosons, the signal production rate is very large because they can come from on-shell $W$ boson decay, but the jets have small transverse momentum in this case. There are complex backgrounds to be considered when detector effects are included. In order to discriminate against hadronic backgrounds at the LHC, more stringent acceptance cuts are applied which restrict the sensitivity of hadron colliders in the low mass region. Without such hadronic backgrounds, lepton colliders have significant advantages for Majorana neutrino detection in the low mass region. Considering different ongoing projects of the next generation lepton colliders [48-51], it is worth examining the potential for Majorana neutrino detection at lepton colliders, which provide a much cleaner environment.

\section{Models and mixing parameters}

We begin our discussion by describing two basic models, to show how Majorana neutrinos fit into the big picture of the SM particles. Notice that the models displayed here do not harm the generality of our phenomenological analysis in section 3 .

First, let us consider the simplest extension, the well known Type I seesaw with $n$ right-handed SM singlet neutrino $N_{a R}(a=1,2, \ldots, n)$. After gauge symmetry breaking, there are Dirac mass terms coming from the normal Yukawa couplings, as well as Majorana mass terms. The full neutrino mass terms can be written as [37]

$$
-\mathcal{L}_{m}^{\nu}=\frac{1}{2}\left(\sum_{a=1}^{3} \sum_{b=1}^{n}\left(\overline{\nu_{a L}} m_{a b}^{\nu} N_{b R}+\overline{N_{b L}^{c}} m_{b a}^{\nu *} \nu_{a R}^{c}\right)+\sum_{b, b^{\prime}=1}^{n} \overline{N_{b L}^{c}} B_{b b^{\prime}} N_{b^{\prime} R}\right)+\text { h.c. }
$$

where $\nu_{a L}(a=1,2,3)$ are three generations of flavour neutrinos defined dynamically with respect to charged leptons. After diagonalization, they transform into

$$
-\mathcal{L}_{m}^{\nu}=\frac{1}{2}\left(\sum_{m=1}^{3} m_{\nu_{m}} \overline{\nu_{m L}} \nu_{m R}^{c}+\sum_{m^{\prime}=4}^{3+n} m_{N_{m^{\prime}}} \overline{N_{m^{\prime} L}^{c}} N_{m^{\prime} R}\right)+\text { h.c. }
$$

with the following mixing relations between flavour and mass eigenstates

$$
\begin{aligned}
\nu_{a L} & =\sum_{m=1}^{3} U_{a m} \nu_{m L}+\sum_{m^{\prime}=4}^{3+n} V_{a m^{\prime}} N_{m^{\prime} L}^{c}, \\
U U^{\dagger}+V V^{\dagger} & =I .
\end{aligned}
$$

In terms of the mass eigenstates, the Lagrangian for the $\mathrm{CC}$ and $\mathrm{NC}$ interactions is

$$
\begin{aligned}
\mathcal{L}= & -\frac{g}{\sqrt{2}} W_{\mu}^{+}\left(\sum_{\ell=e}^{\tau} \sum_{m=1}^{3} U_{\ell m}^{*} \overline{\nu_{m}} \gamma^{\mu} P_{L} \ell\right) \\
& -\frac{g}{\sqrt{2}} W_{\mu}^{+}\left(\sum_{\ell=e}^{\tau} \sum_{m^{\prime}=4}^{3+n} V_{\ell m^{\prime}}^{*} \overline{N_{m^{\prime}}^{c}} \gamma^{\mu} P_{L} \ell\right) \\
& -\frac{g}{2 \cos _{W}} Z_{\mu}\left(\sum_{\ell=e}^{\tau} \sum_{m^{\prime}=4}^{3+n} V_{\ell m^{\prime}}^{*} \overline{N_{m^{\prime}}^{c}} \gamma^{\mu} P_{L} \nu_{\ell}\right)+\text { h.c. }
\end{aligned}
$$


where $P_{L}$ is the left-handed chirality projection operator. As can be seen, $U_{\ell m}$ is the non-unitary version of the PMNS mixing matrix in our example, while $V_{\ell m}$ determines the weight of the heavy Majorana neutrinos in leptonic CC and NC interactions. The latter will be the dominant contribution to the lepton-number violation processes at colliders, since the masses of light neutrinos, namely $\nu_{m}(m=1,2,3)$, are at most $\mathcal{O}(\mathrm{eV})$ [37]. Moreover, the existence of $V_{\ell m}$ is also relevant to many other intriguing topics, such as nonunitarity of the light neutrino mixing matrix as well as lepton flavour violation (LFV) [52-60], lepton nonuniversality and electroweak precision tests [61-66]. Therefore we can derive constraints on the heavy neutrino mass and the mixing elements $V_{\ell m}$ from experimental observations.

Now let us take a look at the decay of the heavy Majorana neutrino. For Majorana neutrinos heavier than the Higgs boson $M_{N}>M_{H}$, the decay modes are to a $W$ or a $Z$ or a Higgs boson plus a corresponding SM lepton. The corresponding partial widths are proportional to $\left|V_{\ell m}\right|^{2}[67]$ :

$$
\begin{aligned}
\Gamma\left(N \rightarrow \ell^{-} W^{+}\right) & =\frac{g^{2}}{64 \pi}\left|V_{\ell N}\right|^{2} \frac{M_{N}^{3}}{M_{W}^{2}}\left(1-\frac{M_{W}^{2}}{M_{N}^{2}}\right)^{2}\left(1+2 \frac{M_{W}^{2}}{M_{N}^{2}}\right) \\
\Gamma\left(N \rightarrow \nu_{\ell} Z\right) & =\frac{g^{2}}{128 \pi}\left|V_{\ell N}\right|^{2} \frac{M_{N}^{3}}{M_{W}^{2}}\left(1-\frac{M_{Z}^{2}}{M_{N}^{2}}\right)^{2}\left(1+2 \frac{M_{Z}^{2}}{M_{N}^{2}}\right) \\
\Gamma\left(N \rightarrow \nu_{\ell} H\right) & =\frac{g^{2}}{128 \pi}\left|V_{\ell N}\right|^{2} \frac{M_{N}^{3}}{M_{W}^{2}}\left(1-\frac{M_{H}^{2}}{M_{N}^{2}}\right)^{2}
\end{aligned}
$$

For Majorana neutrinos lighter than the $W$ boson $M_{N}<M_{W}$, the decay is via charged and neutral current interactions to SM leptons plus pseudoscalar or vector mesons, a detailed list of which can be found in [37]. Adding all the partial decay widths, the total width of the heavy Majorana neutrino is [34]

$$
\Gamma_{N} \approx\left\{\begin{array}{lll}
\sum_{\ell}\left|V_{\ell N}\right|^{2} \frac{G_{F} M_{N}^{3}}{8} & \text { for } & M_{N}>M_{Z}, M_{H} \\
\sum_{\ell}\left|V_{\ell N}\right|^{2} \frac{G_{F}^{2} M_{N}^{5}}{10^{3}} & \text { for } & M_{N} \ll M_{W} .
\end{array}\right.
$$

It is still quite narrow even for $M_{N} \sim 1 \mathrm{TeV}$ with small mixing angles. For $M_{N} \gg$ $M_{W}, M_{Z}, M_{H}$, the decay branching ratios are $\operatorname{Br}\left(\ell^{-} W^{+}\right) \approx \operatorname{Br}\left(\nu_{\ell} Z\right) \approx \operatorname{Br}\left(\nu_{\ell} H\right) \approx 25 \%$.

Another way to incorporate Majorana neutrinos is through $\mathrm{SU}(2)_{L}$ lepton triplets which is known as the Type III Seesaw mechanism [68-70]. Here we employ triplet leptons $\Sigma_{L}$ in the representation $(1,3,0)$ of the SM gauge group [70]:

$$
\Sigma_{L}=\Sigma_{L}^{a} \sigma^{a}=\left(\begin{array}{cc}
\Sigma_{L}^{0} / \sqrt{2} & \Sigma_{L}^{+} \\
\Sigma_{L}^{-} & -\Sigma_{L}^{0} / \sqrt{2}
\end{array}\right), \quad \Sigma_{L}^{ \pm} \equiv \frac{\Sigma_{L}^{1} \mp i \Sigma_{L}^{2}}{\sqrt{2}}, \quad \Sigma_{L}^{0}=\Sigma_{L}^{3},
$$

where $\Sigma_{L}^{ \pm}$have electric charges $Q= \pm 1$, and the $\sigma^{a}(a=1,2,3)$ are the usual Pauli matrices. The relevant Lagrangian for generating neutrinos masses is given by

$$
\mathcal{L}=\frac{1}{2} \operatorname{Tr}\left[\overline{\Sigma_{L}} i \not D \Sigma_{L}\right]-\frac{M_{\Sigma}}{2} \overline{\Sigma_{L}^{0}} \Sigma_{R}^{0 c}-M_{\Sigma} \overline{\Sigma_{L}^{-}} \Sigma_{R}^{+c}-Y_{\Sigma} \bar{L} \Sigma_{R}^{c} i \sigma^{2} H^{*}+\text { h.c. }
$$


where the first three are the kinetic and mass terms of the triplet, and the last one is its Yukawa coupling to the SM left-handed lepton doublet $L$ and Higgs doublet $H$. After gauge symmetry breaking, the Yukawa coupling will bring about mass mixing among the triplet and SM leptons which is key to our process discussed below. In addition to the mixing between charged triplet leptons $\Sigma_{L}^{ \pm}$and SM charged leptons $e, \mu, \tau$, which is special to the Type III Seesaw mechanism, $\Sigma_{R}^{0 c}$ has the same Yukawa coupling and mass term as the right-handed neutrino of the Type I Seesaw above.

After an unitary transformation to the mass basis, we use $N$ and $E^{ \pm}$to denote the mass eigenstates of triplet leptons, then the gauge eigenstates become

$$
\begin{aligned}
\Sigma^{ \pm} & =U E^{ \pm}-\sqrt{2} V \ell_{m}^{ \pm}, & \Sigma^{0}=U N-V \nu_{m} \\
\ell^{ \pm} & =U \ell_{m}^{ \pm}+\sqrt{2} V E^{ \pm}, & \nu=U N+V \nu_{m} \\
\text { with } \quad|U| & \sim \mathcal{O}(1), \quad|V| \sim \frac{Y_{\Sigma} v_{0}}{\sqrt{2} M_{\Sigma}} \ll 1 . &
\end{aligned}
$$

The relevant gauge interaction Lagrangian in the mass basis is

$$
\begin{aligned}
\mathcal{L} \chi_{i}^{0}-\bar{E}\left(e U A_{\mu} \gamma^{\mu}+g \cos \theta_{W} U Z_{\mu} \gamma^{\mu}\right) E-g U \bar{E} W_{\mu}^{-} \gamma^{\mu} N \\
-\frac{g}{2 \cos \theta_{W}} Z_{\mu}\left(V \bar{N} \gamma^{\mu} P_{R} \nu+\sqrt{2} V \bar{E} \gamma^{\mu} P_{R} \ell\right) \\
-g W_{\mu}^{+}\left(V \bar{\nu} \gamma^{\mu} P_{L} E+\frac{1}{\sqrt{2}} V \bar{N} \gamma^{\mu} P_{R} \ell\right)+\text { h.c. }
\end{aligned}
$$

At tree level, the heavy leptons $N$ and $E^{ \pm}$are degenerate in mass, and we have [70]

$$
m_{\nu} \approx \frac{Y_{\Sigma}^{2} v_{0}^{2}}{2 M_{\Sigma}}, \quad M_{N} \approx M_{E} \approx M_{\Sigma}
$$

However, radiative corrections will create a mass splitting and make the charged $E^{ \pm}$slightly heavier than the $N$. So the available decay channels of the heavy Majorana $N$ here are the same as that of the Type I Seesaw [69].

One thing we would like to point out is that a "minimal canonical" Seesaw mechanism is not likely to be the final answer to explain the reality of neutrino mass generation. Besides, the parameter space of the minimal Seesaw mechanisms is often severely restricted and unreachable in current experiments. For example, in the minimal, high-scale Type I Seesaw, we can obtain the following relationship for mixing elements of the Majorana neutrino [70],

$$
\left|V_{\ell N}\right|^{2} \sim \frac{m_{\nu}}{M_{N}}
$$

For a heavy neutrino mass of $M_{N} \sim 100 \mathrm{GeV}$, this implies $\left|V_{\ell N}\right|^{2} \sim 10^{-14}-10^{-12}$, well below any foreseeable experiments can reach. This calls for generalization of the minimal Seesaw models, which would give rise to greater phenomenology. Hybrid Seesaw models are one type of variant in which two or more "canonical" mechanisms are combined. For example, in the Type I+II Hybrid Seesaw model, the light neutrino mass matrix $M_{\nu}$ is given by [70]

$$
M_{\nu}=M_{L}-M_{D} M_{N}^{-1} M_{D}^{T}
$$


Here, $M_{D}, M_{N}$ are respectively the Dirac and Majorana mass matrixes from the Type I model, whereas $M_{L}$ comes from the Type II mechanism. In this scenario, sub-eV neutrino masses can arise not only from parametrically small Type I and II masses but additionally from an incomplete cancellation of the two terms. Hence $\left|V_{\ell N}\right|^{2}$ could break the relation in eq. (2.17), and need not be that small.

Since our goal is to study the detection capability of near future experiments, our analysis in section 3 takes a model-independent phenomenological approach. The heavy Majorana neutrino mass $M_{N}$ and mixing elements $\left|V_{\ell N}\right|$ are treated as free parameters. So far, neutrinoless double- $\beta$ decay $(0 \nu \beta \beta)$ experiments have set the most stringent bound on mixing with electrons $[71-73]$ :

$$
\sum_{N} \frac{\left|V_{e N}\right|^{2}}{m_{N}}<5 \times 10^{-8} \mathrm{GeV}^{-1} .
$$

This makes it difficult for colliders to produce events involving electrons. So instead, we focus on muon relevant signals and seek to improve the detecting sensitivity on $V_{\mu N}$. The most stringent constraint on mixing with muons from LEP2 experiments requires $\left|V_{\mu N}\right|^{2} \lesssim 10^{-4}-10^{-5}$ for $5 \mathrm{GeV}<m_{N}<80 \mathrm{GeV}$ [74-76]. For neutrinos heavier than the $Z$ bosons, there are also plenty of researches based on electron colliders [77-85]. However, due to the limitation of the center-of-mass (c.m.) energy of previous LEP2 experiments, the most stringent constraint for heavy Majorana neutrino masses between 100 and $1600 \mathrm{GeV}$ is from the LHC experiments based on the production via the DY process [42-47]. At very high scales, another production mechanism will come into play, which is the vector boson fusion (VBF) channel $W \gamma \rightarrow N \ell^{ \pm}$, benefiting from its collinear logarithmic $t$-channel enhancement $[86,87]$. It dominates over the DY mechanism for $m_{N} \sim 1 \mathrm{TeV}(770 \mathrm{GeV})$ at the $14 \mathrm{TeV}$ LHC (100 TeV VLHC) [87]. Figure 1 summarizes the current constraints on heavy right-handed neutrino mixing with the muon neutrino [42, 44, 46, 47, 75, 76]. A comprehensive discussion regarding the upper bounds on the mixing elements from all kinds of experimental and prospective studies can be found in [70, 88-91].

\section{Collider signatures}

The "smoking gun" signal for heavy Majorana neutrinos at hadron colliders is the like-sign dilepton final state with two jets and no missing transverse energy: $p p \rightarrow W^{*} \rightarrow N \ell^{ \pm} \rightarrow$ $\ell^{ \pm} \ell^{ \pm} j j[34,37]$. For electron-positron colliders, a similar approach has been proposed to produce lepton-number violation processes [67, 92]:

$$
e^{+} e^{-} \rightarrow N \ell^{ \pm} W^{\mp} \rightarrow \ell^{ \pm} \ell^{ \pm}+4 j
$$

with a final state of a same-sign dilepton pair plus four jets and no missing energy. For the reason mentioned above, we do not hold out much hope that electrons could play a significant role in this process. Hence we limit the dilepton signature in final states to be same-sign dimuon $\mu^{ \pm} \mu^{ \pm}$, and ignore mixing with the electron $\left(V_{e N}=0\right)$, giving the most conservative setting to derive bounds on $V_{\mu N}$. Representative Feynman diagrams 


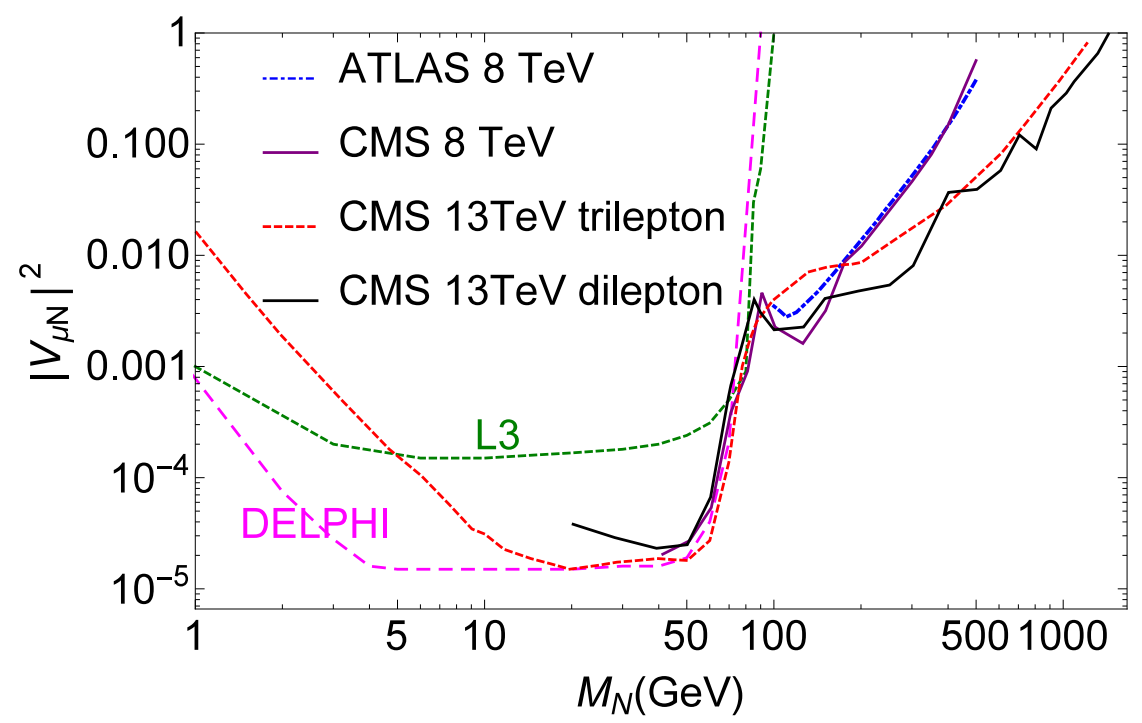

Figure 1. Limits at $95 \% \mathrm{CL}$ on the mixing between the muon neutrino $\nu_{\mu}$ and a single heavy neutrino in the mass range $1 \mathrm{GeV}-1600 \mathrm{GeV}$, from different current collider direct searches: L3 [75], DELPHI [76], ATLAS [42], CMS $8 \mathrm{TeV}$ data [44], and the the trilepton analysis [46] and dilepton analysis [47] based on CMS $13 \mathrm{TeV}$ data.

with dimuon in final states are displayed in figure 2. Channels involving the $e N W$ vertex have been omitted. In these diagrams, a Majorana neutrino can be produced on shell while kinematically accessible, and decays to a muon plus two jets. Due to the resonance enhancement effect, the total production rate is basically proportional to $\left|V_{\mu N}\right|^{2}$. For the Majorana neutrino mass range of our interest, the decay width is small enough so that the narrow-width approximation (NWA) is applicable. Thus the total cross section of our signal process can be approximately broken down as

$$
\begin{aligned}
& \sigma\left(e^{+} e^{-} \rightarrow \mu^{ \pm} \mu^{ \pm}+4 j\right) \approx \\
& \sigma\left(e^{+} e^{-} \rightarrow N \mu^{ \pm}+2 j\right) \operatorname{Br}\left(N \rightarrow \mu^{ \pm}+2 j\right) \equiv S_{\mu \mu} \sigma_{0}
\end{aligned}
$$

where $\sigma_{0}$ is a function of Majorana neutrino mass $m_{N}$, and independent of the mixing parameters when the heavy neutrino decay width is narrow. $S_{\mu \mu}$ is an "effective mixing parameter" of $N$ with a muon, and in the cases of the minimal Type I and Type III Seesaw, is defined as [37]

$$
S_{\mu \mu}=\frac{\left|V_{\mu N}\right|^{4}}{\sum_{\ell=e}^{\tau}\left|V_{\ell N}\right|^{2}}
$$

This enables us to get a direct understanding of the effect of the mixing parameters. We calculate the cross sections of the signal process at the CEPC and the ILC energies. In light of the possibility for a later upgrade of the ILC to $1 \mathrm{TeV}$, both $500 \mathrm{GeV}$ and $1 \mathrm{TeV}$ c.m. energies are considered. The total cross section values for different heavy neutrino masses are shown in figure 3 , assuming a benchmark value $S_{\mu \mu}=10^{-4}$. This is the typical value of the bounds previously simulated at hadron colliders [37]. For $m_{N}<M_{W}$, the cross sections almost stay the same. When the mass approaches the c.m. energy limit, the 


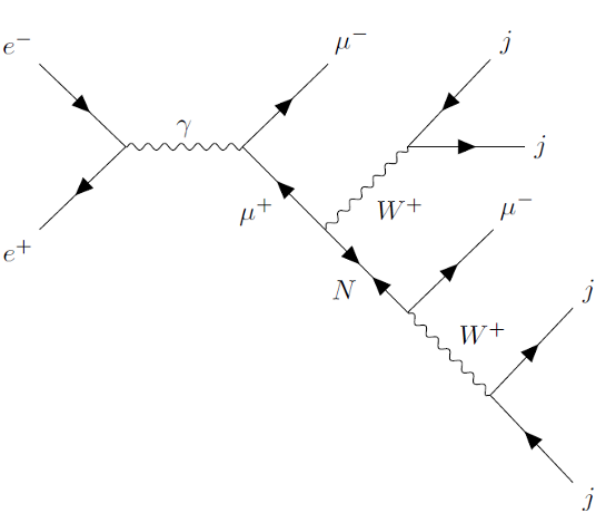

(a)

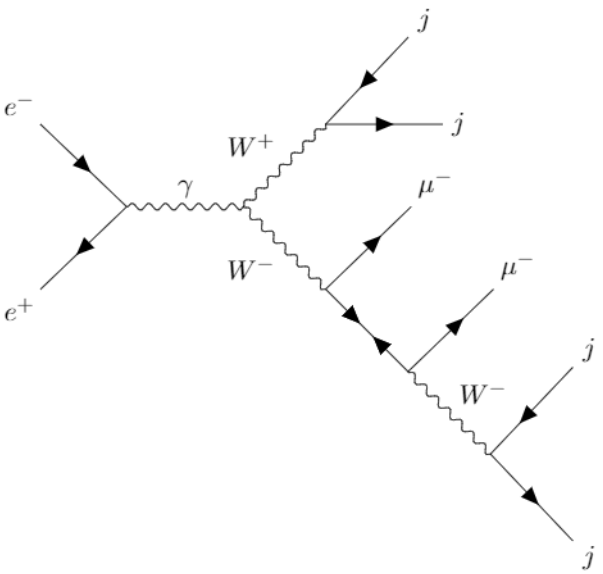

(b)

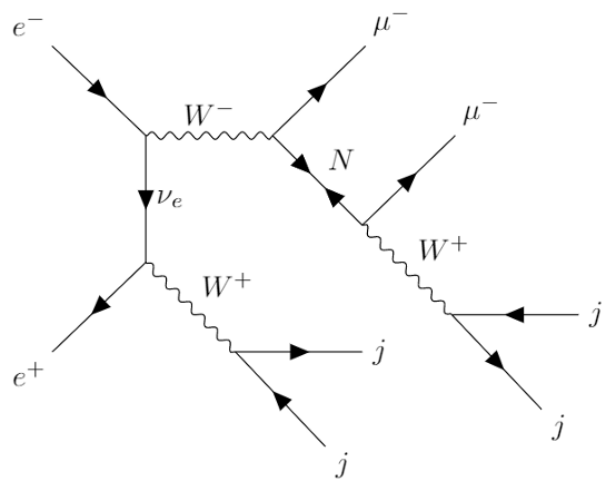

(c)

Figure 2. Three sample Feynman diagrams for the process $e^{+} e^{-} \rightarrow N \mu^{ \pm} W^{\mp} \rightarrow \mu^{ \pm} \mu^{ \pm}+4 j$.

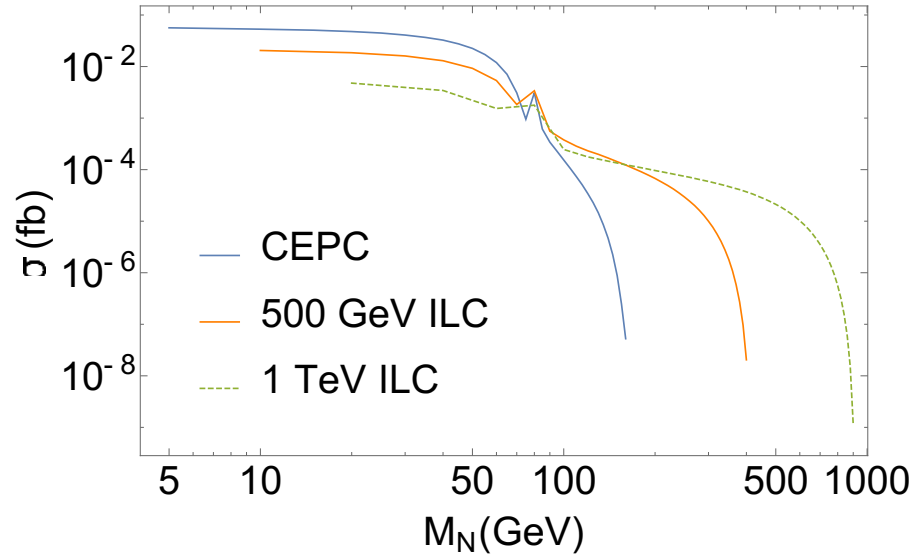

Figure 3. Total cross section for the process $e^{+} e^{-} \rightarrow N \mu^{ \pm} W^{\mp} \rightarrow \mu^{ \pm} \mu^{ \pm}+4 j$ at the CEPC and ILC $(500 \mathrm{GeV}$ and $1 \mathrm{TeV})$, with $S_{\mu \mu}=10^{-4}$. 
cross sections drop sharply. The bumps at approximately $80 \mathrm{GeV}$ correspond to the energy threshold of on-shell production of $W$ bosons in the decay of Majorana neutrinos. As we can see, the CEPC has an advantage in the lower Majorana mass range (about $5-100 \mathrm{GeV}$ ) and the $1 \mathrm{TeV}$ ILC in the larger mass range (about $200-900 \mathrm{GeV}$ ). We will mainly present our results at $1 \mathrm{TeV}$ for the ILC.

\subsection{Phenomenological Analysis at the CEPC}

Based on the discussion in the previous section, we study the Majorana neutrino production and lepton-number violating process at future lepton colliders. The Circular Electron Positron Collider (CEPC) proposed by the Chinese high energy physics community is an ambitious project. Envisioned to operate at $\sqrt{s} \sim 250 \mathrm{GeV}$, CEPC may be able to shed light on Majorana neutrinos with lower mass $\left(m_{N}<100 \mathrm{GeV}\right)$. Compared to linear colliders, it is easier for circular colliders to achieve a much higher integrated luminosity, which amounts to $5 \mathrm{ab}^{-1}$ in the case of CEPC. In this section, we take performance of the CEPC as benchmark for our numerical analysis $[50,51]$. Since the FCC-ee is also planning to run at the $Z H$ peak for almost the same amount of integrated luminosity [48], the analyses below also apply to the FCC-ee case. To reach a high particle identification efficiency, we apply following basic cuts on transverse momentum $\left(p_{T}\right)$ and pseudorapidity $(\eta)$ :

$$
\begin{aligned}
p_{T}(\mu) & >5 \mathrm{GeV}, & \eta(\mu) & <2.5, \\
p_{T}(j) & >5 \mathrm{GeV}, & \eta(j) & <2.5 .
\end{aligned}
$$

To mimic the effect of detector resolution, we smear the four-momentum of the final state particles, following the performance specified in refs. [50, 51]. For muons in the final state, the silicon tracking system provides high track momentum resolution, which performs approximately as

$$
\Delta\left(\frac{1}{p_{T}}\right)=2 \times 10^{-5} \oplus \frac{10^{-3}}{p_{T} \sin \theta} .
$$

The energy resolution of jets measured in the Hadron Calorimeter (HCAL) is

$$
\frac{\delta E}{E}=\frac{0.3}{\sqrt{E / \mathrm{GeV}}} \oplus 0.02 .
$$

To get a detailed understanding of the signal process, we further analyze the kinematical features of the final states. We choose three different masses $m_{4}=25,50 \mathrm{GeV}$ (below $m_{W}$ threshold) and $100 \mathrm{GeV}$ (above $m_{W}$ threshold) for illustration. The key point is that there are two well-isolated same-sign muons, with no missing energy. Therefore we calculate the radial distance $\left(\Delta R=\sqrt{\Delta \eta^{2}+\Delta \phi^{2}}\right)$ of different particle pairs in the final states: $\Delta R_{\ell \ell}, \Delta R_{\ell j}$ and $\Delta R_{j j}$ respectively. After normalization, the distributions of the minimal isolation are shown separately in figure $4(\mathrm{a})$, (b) and (c). Since all particles in the final state are visible in principle, by nature there is neither missing transverse momentum nor missing total energy in the signal process. However, misbalance in the energy-momentum measurement would still result from finite resolution of detectors, which is simulated by the smearing effect in eqs. (3.6)-(3.7). This would be regarded as missing transverse momentum or missing energy. An advantage over hadron colliders is that the c.m. energy at 


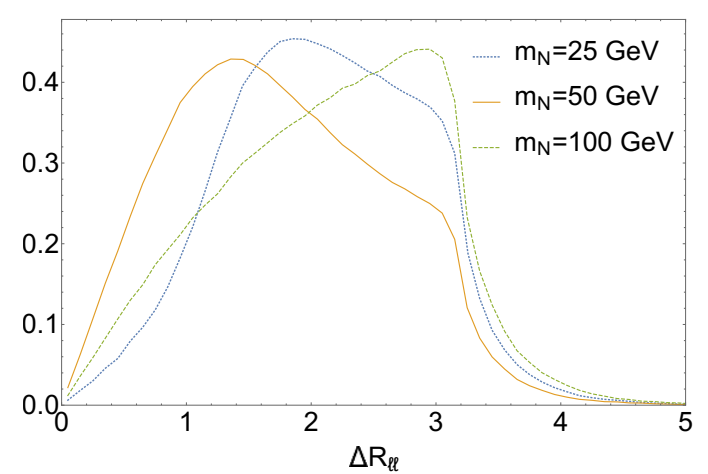

(a)

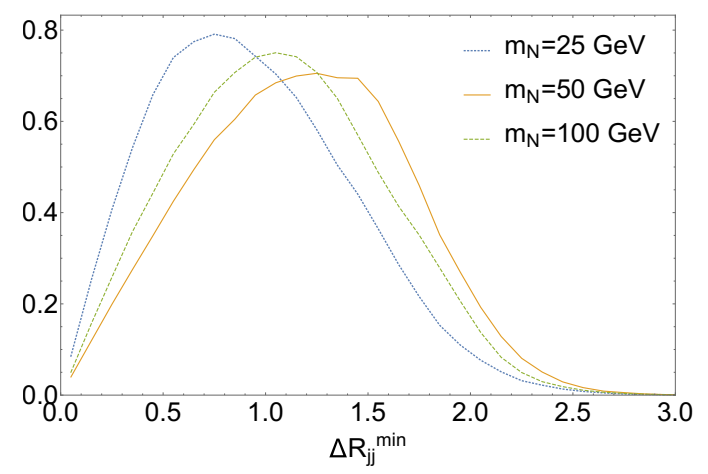

(c)

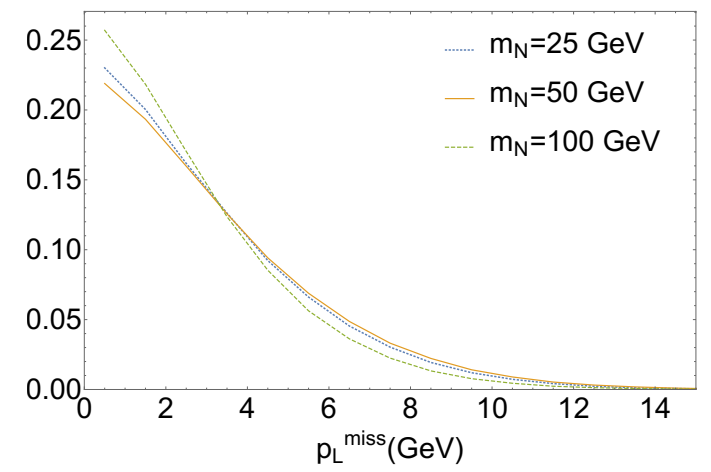

(e)

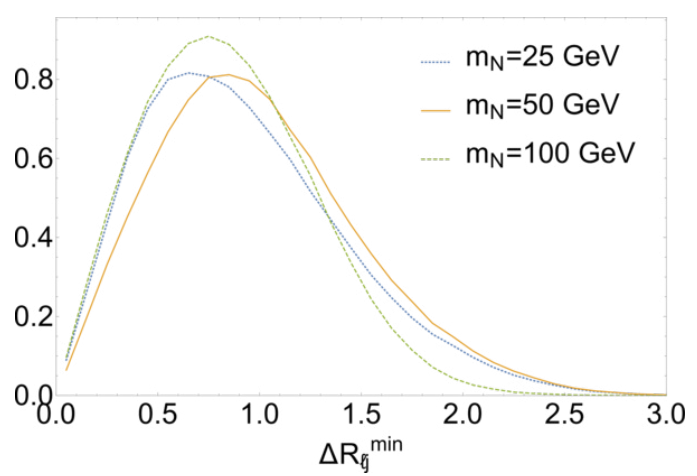

(b)

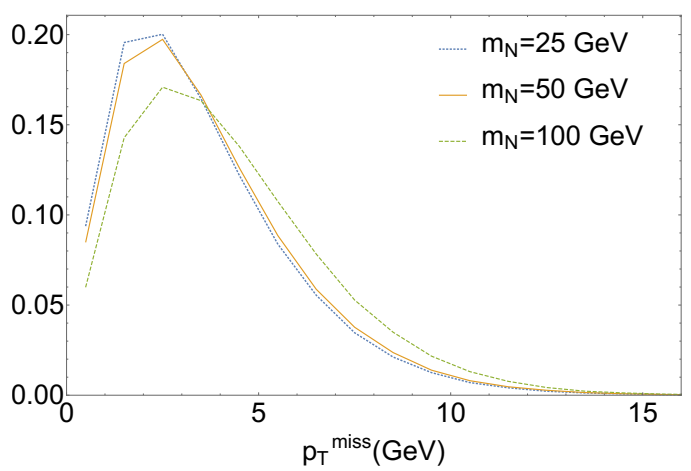

(d)

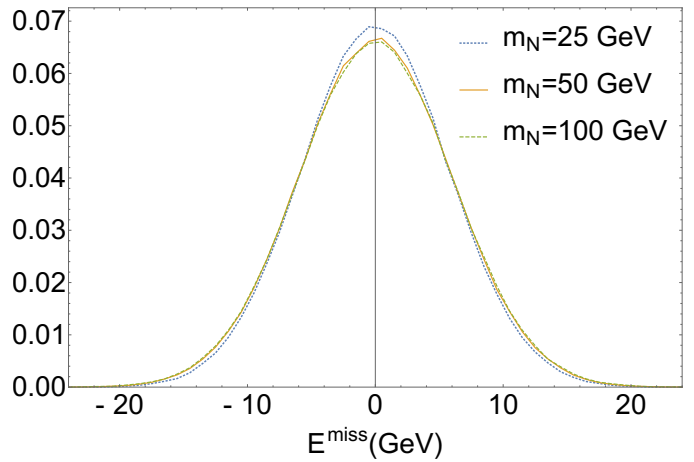

(f)

Figure 4. Normalized distributions $\sigma^{-1} \mathrm{~d} \sigma / \mathrm{d} X$ for $m_{N}=25,50$ and $100 \mathrm{GeV}$ at the CEPC for (a) the radial isolation of muon pairs $\Delta R_{\ell \ell}$; the minimal radial isolation (b) $\Delta R_{\ell j}^{\min }$ and (c) $\Delta R_{j j}^{\min }$; (d) the missing transverse momentum $\not p_{T}$; (e) the missing longitudinal momentum $\not p_{L}$ and (f) the missing total energy $\not \mathbb{E}$. The missing total energy is the c.m. energy $250 \mathrm{GeV}$ minus the sum of energies of all visible particles in the final state.

an $e^{+} e^{-}$collider is precisely measurable and adjustable. We exploit this feature by fully analyzing the missing transverse momentum, missing longitudinal momentum, as well as missing total energy, and the distributions are plotted separately in figure 4(d), (e) and (f). In addition, we emphasize the on-shell production of one Majorana neutrino $N$ and at least one $W^{ \pm}$boson in the signal process. Normally this could be useful for reconstructing 


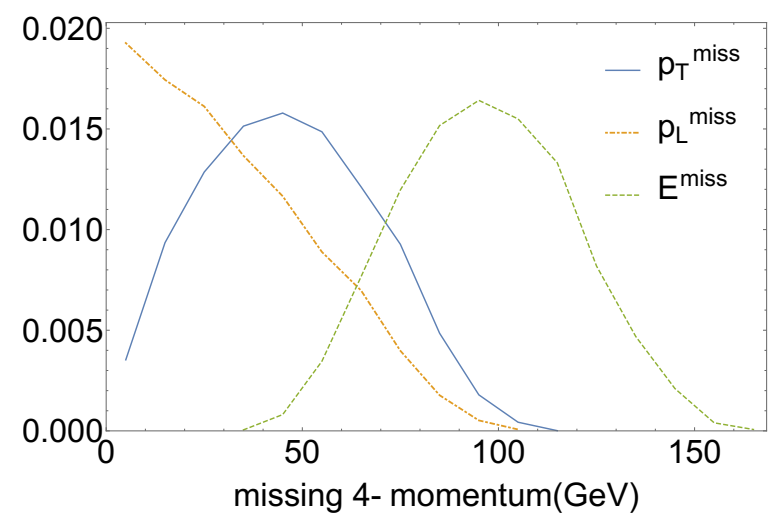

Figure 5. Normalized distributions $\sigma^{-1} \mathrm{~d} \sigma / \mathrm{d} X$ for background channel $e^{-} e^{+} \rightarrow W^{+} Z+4 j, W^{+} \rightarrow$ $\mu^{+}+\nu_{\mu}, Z \rightarrow \mu^{-} \mu^{+}$at the CEPC for the missing transverse momentum $\not p_{T}$, missing longitudinal momentum $\not p_{L}$ and missing total energy $\not \subset$. The missing total energy is the c.m. energy $250 \mathrm{GeV}$ minus the sum of energies of all visible particles in the final state.

particles from invariant masses of final state particles, which are $m(\ell j j)$ in $N$ decay and $m(j j)$ in $W^{ \pm}$decay. However there are extra muon and jets from other sources which would impair this, and as we will see later, the missing momentum cuts already achieve excellent discriminating power. Therefore, the invariant mass cuts are not considered here.

Since the SM Lagrangian conserves lepton number, there is no such process with $\Delta L=2$ in the SM. However, backgrounds to the like-sign dilepton signal can arise when some particles are missing from detection. This comes from $4 \ell+4 j$ final states with two leptons lost in the beam pipe. By generating all processes with such final states in the SM, we find most of them are at the level of $10^{-7} \mathrm{fb}$. What is left outstanding is the channel of diboson plus four jets: $e^{-} e^{+} \rightarrow W Z+4 j$, with the two bosons decaying leptonically. We further analyze the kinematical information of this background channel and find the best discriminating power comes from missing four-momentum. We display the distributions of $\not p_{T}, \not p_{L}$ and $\not$ of the background channel $e^{-} e^{+} \rightarrow W^{+} Z+4 j, W^{+} \rightarrow \mu^{+}+\nu_{\mu}, Z \rightarrow \mu^{-} \mu^{+}$ in figure 5. Comparing to the distributions of signal events, it is easy to see that $p_{T}$ and $\mathbb{E}$ have the best discriminating powers, and could essentially eliminate this background.

Based on the analysis described above, we impose the following cuts to guarantee qualified events:

$$
\begin{aligned}
& \Delta R_{\ell \ell}>0.4, \quad \Delta R_{\ell j}^{\min }>0.4, \quad \Delta R_{j j}^{\min }>0.4, \\
& \not p_{T}<10 \mathrm{GeV}, \quad \quad \notin<20 \mathrm{GeV} \text {. }
\end{aligned}
$$

After imposing the basic cuts in eqs. (3.4)-(3.5) and the selection cuts in eqs. (3.8)-(3.9), all backgrounds are negligibly small. The signal cross sections for different heavy neutrino masses at the CEPC are shown in figure 6 , where we set $S_{\mu \mu}=10^{-4}$ as a benchmark value. The reduction in rate is mainly due to basic acceptance cuts, especially in the low mass range. The cross section reaches its maximum when neutrino mass is about $25 \mathrm{GeV}$, and then drops with increasing $m_{N}$. Although the cross section is only at the level of 


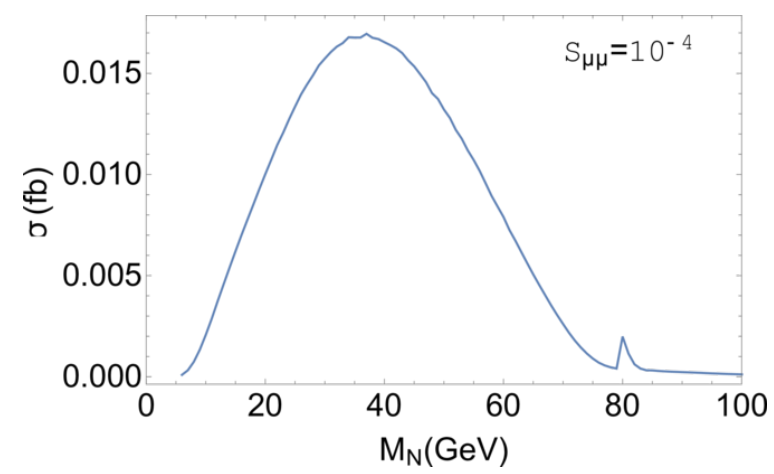

Figure 6. Total cross section for the process $e^{+} e^{-} \rightarrow N \mu^{ \pm} W^{\mp} \rightarrow \mu^{ \pm} \mu^{ \pm}+4 j$ after all the cuts in eqs. (3.4)-(3.5) and eqs. (3.8)-(3.9) at the CEPC, with $S_{\mu \mu}=10^{-4}$.

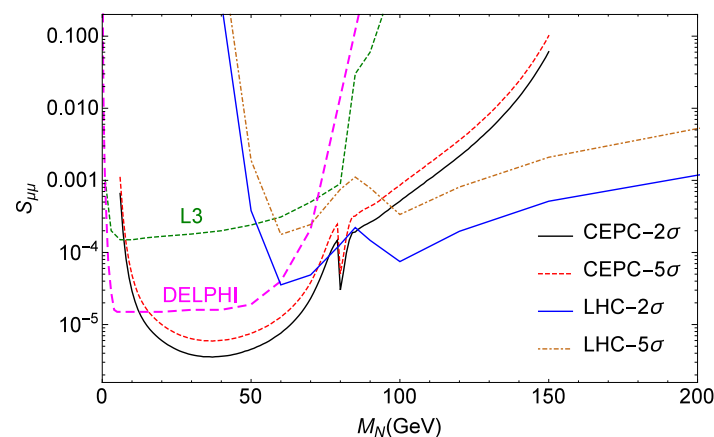

(a)

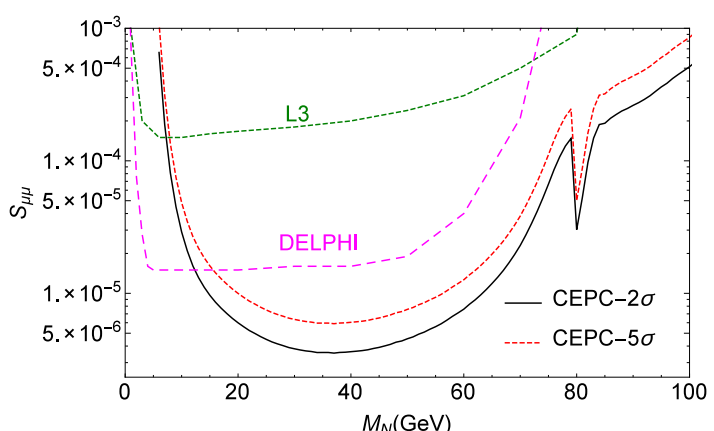

(b)

Figure 7. Sensitivity on $S_{\mu \mu}$ at the CEPC. (b) is an enlarged version of (a) in the range of 0$100 \mathrm{GeV}$. For comparison, the 95\% bound from the L3 [75] and DELPHI [76] searches and simulated LHC results in [37] are presented.

$10^{-2}-10^{-3} \mathrm{fb}$, the high integrated luminosity of the CEPC results in a sufficient number of events produced.

To obtain the signal significance, we consider Poisson statistics for the low event rate. For instance, a $95 \%(2 \sigma)$ bound on the signal for no background would need a signal event rate $N_{S}=\mathcal{L} \sigma_{0} S_{\mu \mu} \geq 3$, where $\mathcal{L}$ is the integrated luminosity. Our final results for the sensitivity on the mixing parameter $S_{\mu \mu}$ are shown in figure 7 together with the L3 [75] and DELPHI [76] bounds from Z decay and the previous simulated LHC results in [37]. Figure 7 (a) displays the mass range of $0-200 \mathrm{GeV}$, and (b) is a detail view of (a) in the range of $0-100 \mathrm{GeV}$. The simulation results at the LHC are based on a c.m. energy of $14 \mathrm{TeV}$ and an integrated luminosity of $100 \mathrm{fb}^{-1}$. We see that there is a significant improvement on detection sensitivity at the CEPC in the mass range of $m_{N}=15-80 \mathrm{GeV}$. Compared to previous results, it improves measurement sensitivity on the mixing parameter $S_{\mu \mu}$ by roughly one order of magnitude in this mass range. For neutrino masses in the range of 30-50 GeV where the CEPC has the best sensitivity, $S_{\mu \mu}$ can be probed to about $3 \times 10^{-6}$ at a $2 \sigma$ level and $7 \times 10^{-6}$ at a $5 \sigma$ level. 


\subsection{Phenomenological analysis at the ILC}

Since an $e^{+} e^{-}$linear collider has the flexibility to operate at different energies and in different running conditions, experimental programs for the ILC are envisioned at a series of energies well adapted to individual physics goals. The exact run plan that will be carried out at the ILC will depend on the situation at the time of the ILC operation, taking into account new discoveries and measurements from the LHC running. For definiteness in our numerical simulation, we adopt a canonical program with c.m. energy of $1 \mathrm{TeV}$ and integrated luminosity $1000 \mathrm{fb}^{-1}$ [49]. The proposed future lepton colliders in general have similar detector performance and therefore we apply the same basic acceptance cuts as that of CEPC on transverse momentum $\left(p_{T}\right)$, pseudorapidity $(\eta)$, as well as the same smearing effects, which are

$$
\begin{array}{rlrl}
p_{T}(\mu) & >5 \mathrm{GeV}, & \eta(\mu)<2.5, \\
p_{T}(j) & >5 \mathrm{GeV}, \quad & \eta(j)<2.5 . \\
\Delta\left(\frac{1}{p_{T}}\right) & =2 \times 10^{-5} \oplus \frac{10^{-3}}{p_{T} \sin \theta}, \\
\frac{\delta E}{E} & =\frac{0.3}{\sqrt{E / \mathrm{GeV}}} \oplus 0.02 .
\end{array}
$$

Following a similar approach, we analyze the kinematical features of the final state particles and present their distributions. Since the ILC is capable of producing much more massive Majorana neutrinos, we choose $m_{N}=100,200 \mathrm{GeV}$ and $500 \mathrm{GeV}$ as examples for illustration. After normalization, the distributions of the minimal isolation of different particle pairs in the final state: $\Delta R_{\ell \ell}, \Delta R_{\ell j}^{\min }$ and $\Delta R_{j j}^{\min }$, are shown separately in figure 8(a), (b) and (c). And figure 8(d), (e) and (f) are respectively the distributions of the missing transverse momentum $\not p_{T}$, missing longitudinal momentum $\not p_{L}$ and missing total energy $\mathbb{E}$ incurred by finite resolution of the detectors.

At $1 \mathrm{TeV}$ c.m. energy, a significant background comes from production of four on-shell $W$ bosons, with two like-sign $W$ bosons decaying leptonically and the other two decaying hadronically:

$$
e^{-} e^{+} \rightarrow W^{+} W^{+} W^{-} W^{-} \rightarrow \mu^{ \pm} \mu^{ \pm} \nu \nu+4 j
$$

The same final state can be produced via the process

$$
e^{-} e^{+} \rightarrow W^{ \pm} W^{ \pm} W^{\mp} j j \rightarrow \mu^{ \pm} \mu^{ \pm} \nu \nu+4 j
$$

We also analysed other possibilities of four on-shell gauge bosons production, for example

$$
e^{-} e^{+} \rightarrow Z Z W^{+} W^{-} \rightarrow \mu^{ \pm} \mu^{ \pm} \mu^{\mp} \nu+4 j
$$

Because of the presence of two missing leptons (neutrinos or charged muons), these channels can be effectively suppressed by appropriate cuts on missing energy. As an illustration, we plot the distributions of $\not p_{T}, \not p_{L}$ and $\mathbb{E}$ of the four $W$ bosons channel in figure 9 . 


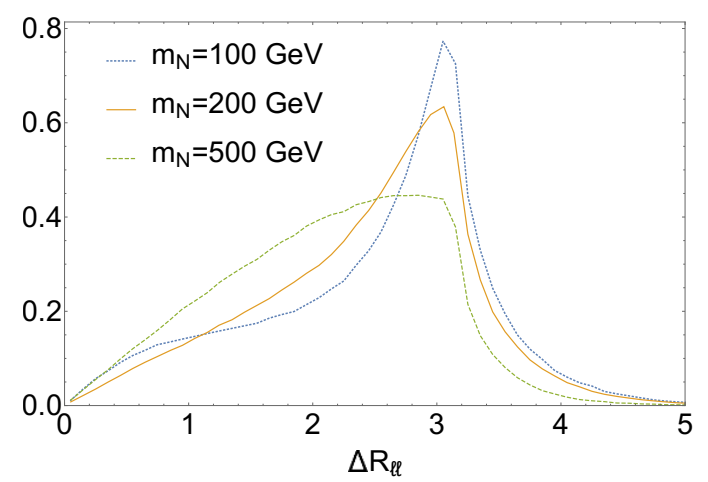

(a)

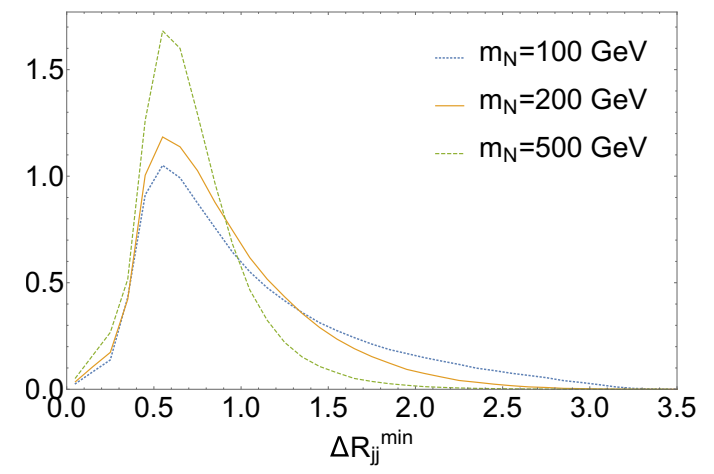

(c)

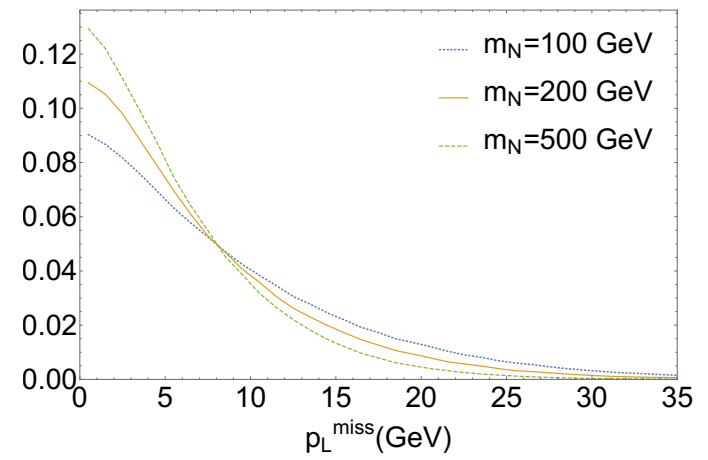

(e)

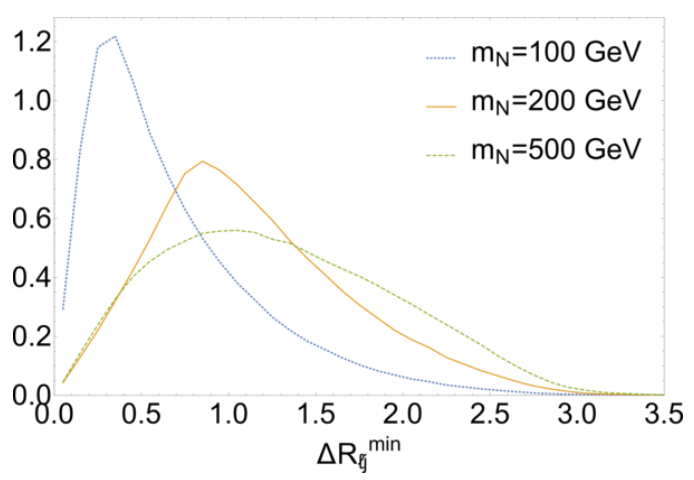

(b)

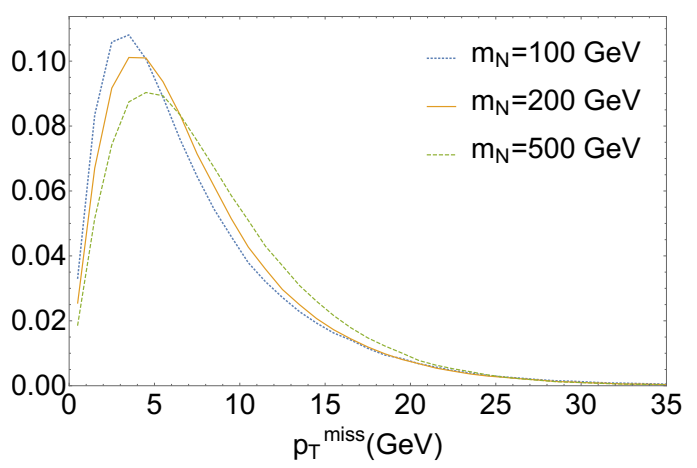

(d)

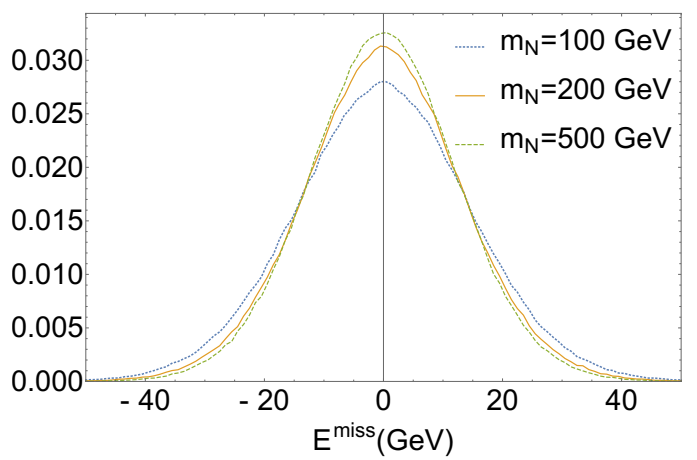

(f)

Figure 8. Normalized distributions $\sigma^{-1} \mathrm{~d} \sigma / \mathrm{d} X$ for $m_{N}=100,200$ and $500 \mathrm{GeV}$ at the $1 \mathrm{TeV}$ ILC for (a) the radial isolation of muon pairs $\Delta R_{\ell \ell}$; the minimal radial isolation (b) $\Delta R_{\ell j}^{\min }$ and (c) $\Delta R_{j j}^{\text {min }}$; (d) the missing transverse momentum $\not p_{T} ;$ (e) the missing longitudinal momentum $\not p_{L}$ and (f) the missing total energy $\mathbb{E}$. The missing total energy is the c.m. energy $1000 \mathrm{GeV}$ minus the sum of energies of all visible particles in the final state. 


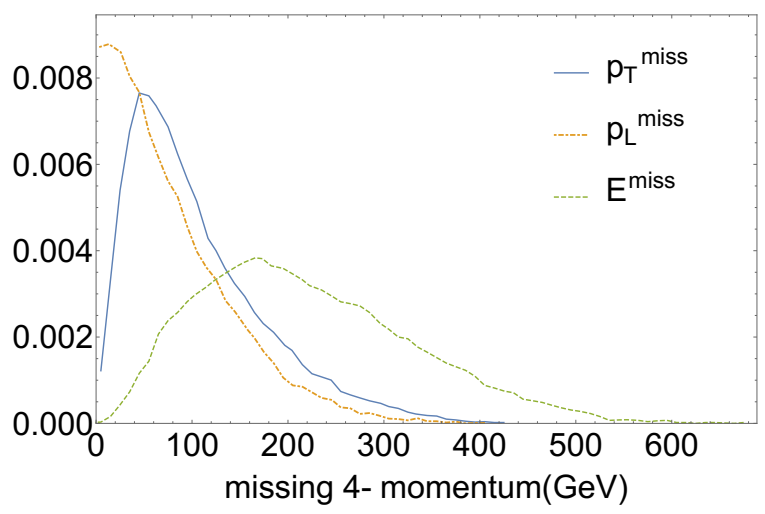

Figure 9. Normalized distributions $\sigma^{-1} \mathrm{~d} \sigma / \mathrm{d} X$ for background channel $e^{-} e^{+} \rightarrow W^{+} W^{+} W^{-} W^{-}$ $\rightarrow \mu^{ \pm} \mu^{ \pm} \nu \nu+4 j$ at the $1 \mathrm{TeV}$ ILC for the missing transverse momentum $\not p_{T}$, missing longitudinal momentum $\not p_{L}$ and missing total energy $\not \mathbb{E}$. The missing total energy is the c.m. energy $1000 \mathrm{GeV}$ minus the sum of energies of all visible particles in the final state.

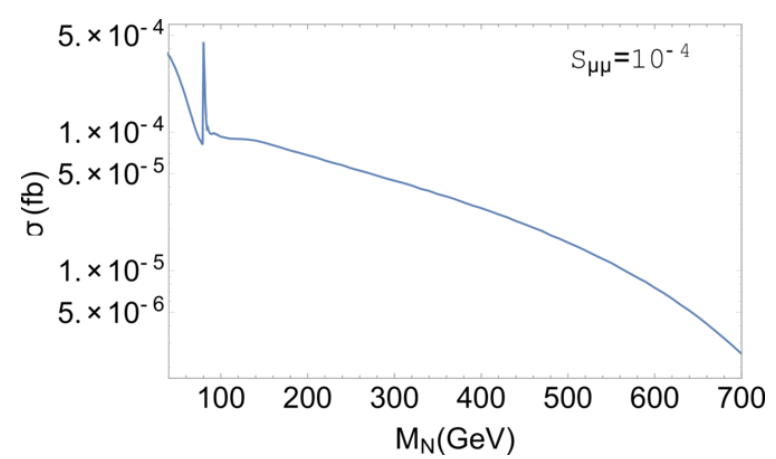

Figure 10. Total cross section for the process $e^{+} e^{-} \rightarrow N \mu^{ \pm} W^{\mp} \rightarrow \mu^{ \pm} \mu^{ \pm}+4 j$ after all the cuts in eqs. (3.10)-(3.11) and eqs. (3.17)-(3.18) at the $1 \mathrm{TeV}$ ILC, with $S_{\mu \mu}=10^{-4}$.

Beyond the basic acceptance cuts, we impose the following additional cuts on radial distance and missing four-momentum to guarantee qualified events:

$$
\begin{aligned}
& \Delta R_{\ell \ell}>0.4, \quad \Delta R_{\ell j}^{\min }>0.4, \quad \Delta R_{j j}^{\min }>0.4, \\
& \not p_{T}<20 \mathrm{GeV}, \quad \not \notin<40 \mathrm{GeV} \text {. }
\end{aligned}
$$

After implementing all the cuts in eqs. (3.10)-(3.11) and eqs. (3.17)-(3.18), the SM background is essentially eliminated and no remaining events survive for the expected luminosity of $1000 \mathrm{fb}^{-1}$ at the ILC. The signal cross sections for different heavy neutrino masses at the ILC are shown in figure 10 , where we set $S_{\mu \mu}=10^{-4}$ as a benchmark value. The reduction in rate is mainly due to basic acceptance cuts, and the cross section gradually drops with increasing $m_{N}$.

We adopt Poisson statistics to determine signal significance and our final results for the sensitivity on the mixing parameter $S_{\mu \mu}$ are shown in figure 11, compared to previous results from the L3 [75] and DELPHI [76] searches and the LHC simulations in [37]. Figure 11(a) displays the mass range of $0-700 \mathrm{GeV}$, and (b) is a detail view of (a) in the range of 200$700 \mathrm{GeV}$. We see that because of its higher c.m. energy, the ILC can improve measurement 


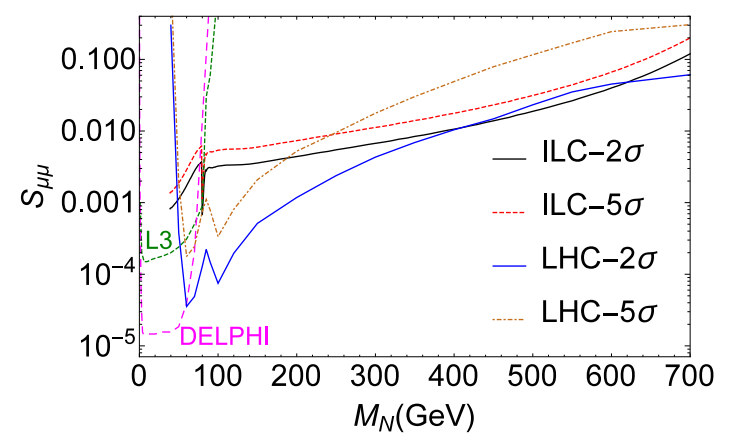

(a)

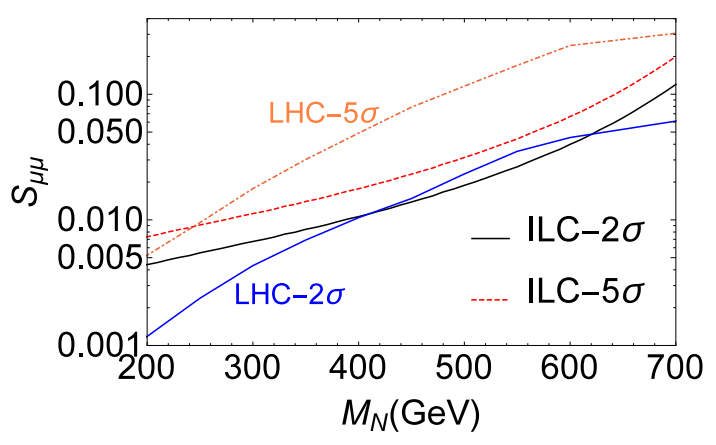

(b)

Figure 11. Sensitivity on $S_{\mu \mu}$ at the ILC. (b) is an enlarged version of (a) in the range of 200$700 \mathrm{GeV}$. For comparison, the 95\% bound from the L3 [75] and DELPHI [76] searches and simulated LHC results in [37] are included.

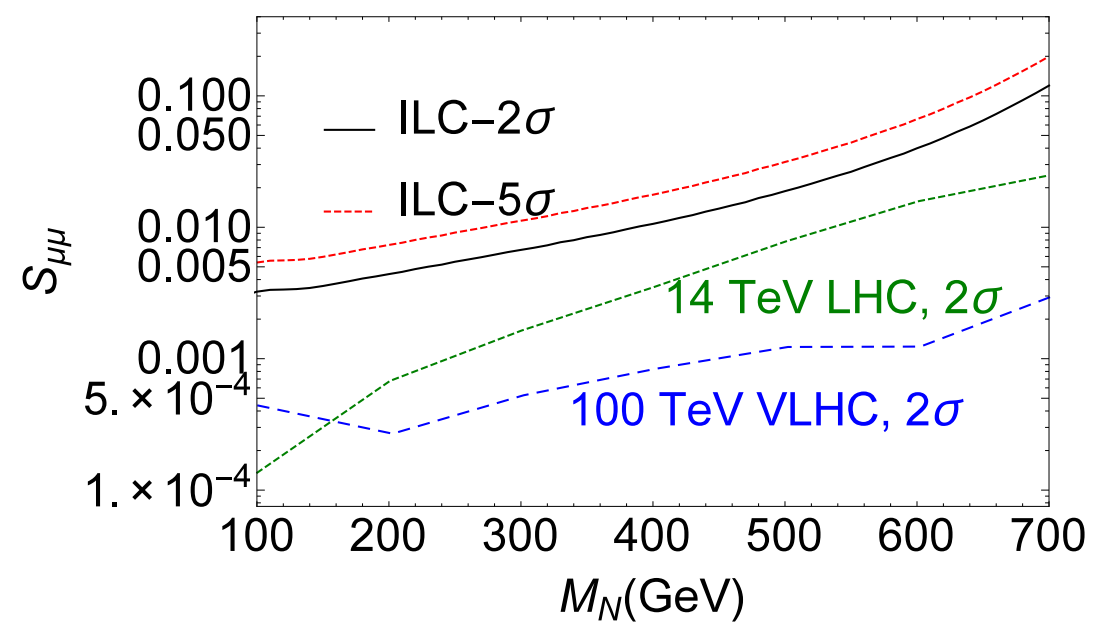

Figure 12. Sensitivity on $S_{\mu \mu}$ at the ILC, compared to the updated result at $14 \mathrm{TeV}$ LHC and the new estimation at $100 \mathrm{TeV}$ VLHC [87].

sensitivity on the mixing parameter $S_{\mu \mu}$ for more massive Majorana neutrinos. At a $5 \sigma$ level, the ILC improves sensitivity by about one order of magnitude for masses between 400 and $600 \mathrm{GeV}$. At a $2 \sigma$ level, there is also a slight amount of improvement in the mass range of $450-600 \mathrm{GeV}$.

As mentioned before, for large Majorana neutrino masses, the $W \gamma$ fusion channel will become important at hadron colliders for contributing lepton number violating events. An estimation of the discovery potential at the $100 \mathrm{TeV}$ VLHC is carried out in [87], combining this new $W \gamma$ fusion channel, the DY process and its next-to-next-to-leading order (NNLO) QCD corrections. The old result of the $14 \mathrm{TeV}$ LHC in [37] has also been updated in [87], where the NNLO corrections are considered and different events analysis is employed. We display the new results in comparison to our ILC estimation in figure 12. The integrated luminosity assumed for the simulation results at the $14 \mathrm{TeV}$ LHC and $100 \mathrm{TeV}$ VLHC in figure 12 is $100 \mathrm{fb}^{-1}$. We can see that the ILC has completely lost its advantage compared to the new results, and higher collision energy is obviously better for very large neutrino masses. 


\section{Summary and conclusions}

In this work, we study a production channel of Majorana neutrinos at lepton colliders that violates lepton number by two units $(\Delta L=2): e^{+} e^{-} \rightarrow N \mu^{ \pm} W^{\mp} \rightarrow \mu^{ \pm} \mu^{ \pm}+4 j$. This process is of profound importance for understanding the nature of neutrino masses. Although the cross section for the signal process is relatively small, lepton colliders still have significant ability to search for signals of Majorana neutrinos due to the clean environment and high integrated luminosity of the next generation lepton colliders. At the CEPC, the mixing parameter $S_{\mu \mu}$ can be probed to about $3 \times 10^{-6}$ at a $2 \sigma$ level and $7 \times 10^{-6}$ at a $5 \sigma$ level in the neutrino mass range of $30-50 \mathrm{GeV}$. At the ILC, which has a comparative advantage in the larger mass range, the measurement sensitivity on $S_{\mu \mu}$ can be improved in the range of $450-600 \mathrm{GeV}$, although future hadron colliders with higher energy and luminosity are obviously more powerful. In summary, in the mass ranges of 30-50 GeV and $450-600 \mathrm{GeV}$, future generation lepton colliders have the potential to make progress on searching for Majorana neutrinos.

Open Access. This article is distributed under the terms of the Creative Commons Attribution License (CC-BY 4.0), which permits any use, distribution and reproduction in any medium, provided the original author(s) and source are credited.

\section{References}

[1] V. Barger, D. Marfatia and K. Whisnant, Progress in the physics of massive neutrinos, Int. J. Mod. Phys. E 12 (2003) 569 [hep-ph/0308123] [INSPIRE].

[2] Particle Data Group, Review of particle physics, Chin. Phys. C 40 (2016) 100001.

[3] P. Minkowski, $\mu \rightarrow e \gamma$ at a rate of one out of $10^{9}$ muon decays?, Phys. Lett. B 67 (1977) 421.

[4] H. Fritzsch and P. Minkowski, Unified interactions of leptons and hadrons, Annals Phys. 93 (1975) 193 [INSPIRE].

[5] R.N. Mohapatra and G. Senjanović, Neutrino mass and spontaneous parity nonconservation, Phys. Rev. Lett. 44 (1980) 912 [INSPIRE].

[6] T. Yanagida, Horizontal gauge symmetry and masses of neutrinos, Conf. Proc. C 7902131 (1979) 95 [INSPIRE].

[7] M. Gell-Mann, P. Ramond and R. Slansky, Complex spinors and unified theories, Conf. Proc. C 790927 (1979) 315 [arXiv:1306.4669] [INSPIRE].

[8] J. Schechter and J.W.F. Valle, Neutrino masses in $\mathrm{SU}(2) \times \mathrm{U}(1)$ theories, Phys. Rev. D 22 (1980) 2227 [INSPIRE].

[9] J.C. Pati and A. Salam, Lepton number as the fourth color, Phys. Rev. D 10 (1974) 275 [Erratum ibid. D 11 (1975) 703] [INSPIRE].

[10] R.N. Mohapatra and J.C. Pati, A natural left-right symmetry, Phys. Rev. D 11 (1975) 2558 [INSPIRE].

[11] G. Senjanović and R.N. Mohapatra, Exact left-right symmetry and spontaneous violation of parity, Phys. Rev. D 12 (1975) 1502 [INSPIRE]. 
[12] J.A. Harvey, D.B. Reiss and P. Ramond, Mass relations and neutrino oscillations in an SO(10) model, Nucl. Phys. B 199 (1982) 223 [InSPIRE].

[13] S. Dimopoulos, L.J. Hall and S. Raby, A predictive framework for fermion masses in supersymmetric theories, Phys. Rev. Lett. 68 (1992) 1984 [INSPIRE].

[14] L.J. Hall and S. Raby, A complete supersymmetric SO(10) model, Phys. Rev. D 51 (1995) 6524 [hep-ph/9501298] [INSPIRE].

[15] I. Dorsner and P. Fileviez Perez, Unification without supersymmetry: neutrino mass, proton decay and light leptoquarks, Nucl. Phys. B 723 (2005) 53 [hep-ph/0504276] [INSPIRE].

[16] I. Dorsner, P. Fileviez Perez and R. Gonzalez Felipe, Phenomenological and cosmological aspects of a minimal GUT scenario, Nucl. Phys. B 747 (2006) 312 [hep-ph/0512068] [INSPIRE].

[17] P. Fileviez Perez, Upper bound on the proton lifetime and the minimal non-SUSY grand unified theory, AIP Conf. Proc. 903 (2006) 385 [hep-ph/0606279] [INSPIRE].

[18] I. Dorsner, P. Fileviez Perez and G. Rodrigo, Fermion masses and the UV cutoff of the minimal realistic SU(5), Phys. Rev. D 75 (2007) 125007 [hep-ph/0607208] [InSPIRE].

[19] A. Zee, Charged scalar field and quantum number violations, Phys. Lett. B 161 (1985) 141.

[20] E. Ma and U. Sarkar, Neutrino masses and leptogenesis with heavy Higgs triplets, Phys. Rev. Lett. 80 (1998) 5716 [hep-ph/9802445] [INSPIRE].

[21] E. Ma and G. Rajasekaran, Softly broken $A_{4}$ symmetry for nearly degenerate neutrino masses, Phys. Rev. D 64 (2001) 113012 [hep-ph/0106291] [INSPIRE].

[22] E. Ma, Pattern of the approximate mass degeneracy of Majorana neutrinos, Mod. Phys. Lett. A 17 (2002) 289 [hep-ph/0201225] [INSPIRE].

[23] K.S. Babu, E. Ma and J.W.F. Valle, Underlying $A_{4}$ symmetry for the neutrino mass matrix and the quark mixing matrix, Phys. Lett. B 552 (2003) 207 [hep-ph/0206292] [INSPIRE].

[24] E. Ma, Plato's fire and the neutrino mass matrix, Mod. Phys. Lett. A 17 (2002) 2361 [hep-ph/0211393] [INSPIRE].

[25] R. Barbier et al., R-parity violating supersymmetry, Phys. Rept. 420 (2005) 1 [hep-ph/0406039] [INSPIRE].

[26] N. Arkani-Hamed, S. Dimopoulos, G.R. Dvali and J. March-Russell, Neutrino masses from large extra dimensions, Phys. Rev. D 65 (2001) 024032 [hep-ph/9811448] [INSPIRE].

[27] Y. Grossman and M. Neubert, Neutrino masses and mixings in non-factorizable geometry, Phys. Lett. B 474 (2000) 361 [hep-ph/9912408] [INSPIRE].

[28] K.R. Dienes and I. Sarcevic, Neutrino flavor oscillations without flavor mixing angles, Phys. Lett. B 500 (2001) 133 [hep-ph/0008144] [InSPIRE].

[29] W.-Y. Keung and G. Senjanović, Majorana Neutrinos and the production of the right-handed charged gauge boson, Phys. Rev. Lett. 50 (1983) 1427 [INSPIRE].

[30] D.A. Dicus, D.D. Karatas and P. Roy, Lepton nonconservation at supercollider energies, Phys. Rev. D 44 (1991) 2033 [INSPIRE].

[31] A. Datta, M. Guchait and A. Pilaftsis, Probing lepton number violation via Majorana neutrinos at hadron supercolliders, Phys. Rev. D 50 (1994) 3195 [hep-ph/9311257] [INSPIRE]. 
[32] F.M.L. Almeida Jr. et al., , On a signature for heavy Majorana neutrinos in hadronic collisions, Phys. Rev. D 62 (2000) 075004 [hep-ph/0002024] [INSPIRE].

[33] O. Panella, M. Cannoni, C. Carimalo and Y.N. Srivastava, Signals of heavy Majorana neutrinos at hadron colliders, Phys. Rev. D 65 (2002) 035005 [hep-ph/0107308] [INSPIRE].

[34] T. Han and B. Zhang, Signatures for Majorana neutrinos at hadron colliders, Phys. Rev. Lett. 97 (2006) 171804 [hep-ph/0604064] [inSPIRE].

[35] S. Bray, J.S. Lee and A. Pilaftsis, Resonant CP-violation due to heavy neutrinos at the LHC, Nucl. Phys. B 786 (2007) 95 [hep-ph/0702294] [INSPIRE].

[36] F. del Aguila, J.A. Aguilar-Saavedra and R. Pittau, Heavy neutrino signals at large hadron colliders, JHEP 10 (2007) 047 [hep-ph/0703261] [INSPIRE].

[37] A. Atre, T. Han, S. Pascoli and B. Zhang, The search for heavy Majorana neutrinos, JHEP 05 (2009) 030 [arXiv:0901.3589] [inSPIRE].

[38] A. Das and N. Okada, Inverse seesaw neutrino signatures at the LHC and ILC, Phys. Rev. D 88 (2013) 113001 [arXiv:1207.3734] [INSPIRE].

[39] J. Gluza and T. Jeliński, Heavy neutrinos and the $p p \rightarrow l l j j$ CMS data, Phys. Lett. B 748 (2015) 125 [arXiv: 1504.05568] [INSPIRE].

[40] J. Gluza, T. Jelinski and R. Szafron, Lepton number violation and 'Diracness' of massive neutrinos composed of Majorana states, Phys. Rev. D 93 (2016) 113017 [arXiv:1604.01388] [INSPIRE].

[41] C. Guo, S.-Y. Guo, Z.-L. Han, B. Li and Y. Liao, Hunting for heavy Majorana neutrinos with lepton number violating signatures at LHC, JHEP 04 (2017) 065 [arXiv:1701.02463] [INSPIRE].

[42] ATLAS collaboration, Search for heavy Majorana neutrinos with the ATLAS detector in pp collisions at $\sqrt{s}=8 \mathrm{TeV}, \mathrm{JHEP} 07$ (2015) 162 [arXiv:1506.06020] [INSPIRE].

[43] CMS collaboration, Search for heavy Majorana neutrinos in $\mu^{ \pm} \mu^{ \pm}+$jets and $e^{ \pm} e^{ \pm}+$jets events in pp collisions at $\sqrt{s}=7$ TeV, Phys. Lett. B 717 (2012) 109 [arXiv:1207.6079] [INSPIRE].

[44] CMS collaboration, Search for heavy Majorana neutrinos in $\mu^{ \pm} \mu^{ \pm}+$jets events in proton-proton collisions at $\sqrt{s}=8 \mathrm{TeV}$, Phys. Lett. B 748 (2015) 144 [arXiv:1501.05566] [INSPIRE].

[45] CMS collaboration, Search for heavy Majorana neutrinos in $e^{ \pm} e^{ \pm}+$jets and $e^{ \pm} \mu^{ \pm}+$jets events in proton-proton collisions at $\sqrt{s}=8 \mathrm{TeV}$, JHEP 04 (2016) 169 [arXiv:1603.02248] [INSPIRE].

[46] CMS collaboration, Search for heavy neutral leptons in events with three charged leptons in proton-proton collisions at $\sqrt{s}=13$ TeV, Phys. Rev. Lett. 120 (2018) 221801 [arXiv: 1802.02965] [INSPIRE].

[47] CMS collaboration, Search for heavy Majorana neutrinos in same-sign dilepton channels in proton-proton collisions at $\sqrt{s}=13 \mathrm{TeV}$, arXiv:1806.10905 [INSPIRE].

[48] M. Benedikt et al., Future Circular Collider Conceptual design report Vol. 2, CERN-ACC-2018-0057 (2018).

[49] T. Behnke et al., The International Linear Collider technical design report - Volume 1: executive summary, arXiv:1306.6327 [INSPIRE]. 
[50] CEPC-SPPC Study Group, CEPC-SPPC preliminary conceptual design report. 1. Physics and detector, IHEP-CEPC-DR-2015-01 (2015).

[51] CEPC Study Group collaboration, CEPC conceptual design report: volume 2 - Physics Es detector, arXiv:1811.10545 [INSPIRE].

[52] S. Antusch et al., Unitarity of the leptonic mixing matrix, JHEP 10 (2006) 084 [hep-ph/0607020] [INSPIRE].

[53] A. Abada et al., Low energy effects of neutrino masses, JHEP 12 (2007) 061 [arXiv: 0707.4058] [INSPIRE].

[54] M. Malinsky, T. Ohlsson and H. Zhang, Non-unitarity effects in a realistic low-scale seesaw model, Phys. Rev. D 79 (2009) 073009 [arXiv:0903.1961] [InSPIRE].

[55] M. Malinsky, T. Ohlsson, Z.-z. Xing and H. Zhang, Non-unitary neutrino mixing and CP-violation in the minimal inverse seesaw model, Phys. Lett. B 679 (2009) 242 [arXiv: 0905.2889] [INSPIRE].

[56] P.S.B. Dev and R.N. Mohapatra, TeV scale inverse seesaw in $\mathrm{SO}(10)$ and leptonic non-unitarity effects, Phys. Rev. D 81 (2010) 013001 [arXiv:0910.3924] [INSPIRE].

[57] D.V. Forero, S. Morisi, M. Tortola and J.W.F. Valle, Lepton flavor violation and non-unitary lepton mixing in low-scale type-I seesaw, JHEP 09 (2011) 142 [arXiv:1107.6009] [INSPIRE].

[58] R. Lal Awasthi and M.K. Parida, Inverse seesaw mechanism in nonsupersymmetric $\mathrm{SO}(10)$, proton lifetime, nonunitarity effects and a low-mass $Z^{\prime}$ boson, Phys. Rev. D 86 (2012) 093004 [arXiv: 1112.1826] [INSPIRE].

[59] D.N. Dinh, A. Ibarra, E. Molinaro and S.T. Petcov, The $\mu$-e conversion in nuclei, $\mu \rightarrow e \gamma, \mu \rightarrow 3 e$ Decays and TeV scale see-saw scenarios of neutrino mass generation, JHEP 08 (2012) 125 [Erratum ibid. 09 (2013) 023] [arXiv: 1205.4671] [INSPIRE].

[60] P. Humbert, M. Lindner and J. Smirnov, The inverse seesaw in conformal electro-weak symmetry breaking and phenomenological consequences, JHEP 06 (2015) 035 [arXiv: 1503.03066] [INSPIRE].

[61] F. del Aguila, J. de Blas and M. Pérez-Victoria, Effects of new leptons in electroweak precision data, Phys. Rev. D 78 (2008) 013010 [arXiv: 0803.4008] [INSPIRE].

[62] E. Akhmedov et al., Improving electro-weak fits with TeV-scale sterile neutrinos, JHEP 05 (2013) 081 [arXiv: 1302.1872] [INSPIRE].

[63] L. Basso, O. Fischer and J.J. van der Bij, Precision tests of unitarity in leptonic mixing, EPL 105 (2014) 11001 [arXiv:1310.2057] [InSPIRE].

[64] S. Antusch and O. Fischer, Non-unitarity of the leptonic mixing matrix: Present bounds and future sensitivities, JHEP 10 (2014) 094 [arXiv:1407.6607] [INSPIRE].

[65] S. Antusch and O. Fischer, Testing sterile neutrino extensions of the Standard Model at future lepton colliders, JHEP 05 (2015) 053 [arXiv: 1502.05915] [INSPIRE].

[66] S. Antusch and O. Fischer, Probing the nonunitarity of the leptonic mixing matrix at the CEPC, Int. J. Mod. Phys. A 31 (2016) 1644006 [arXiv:1604.00208] [inSPIRE].

[67] S. Banerjee et al., Prospects of heavy neutrino searches at future lepton colliders, Phys. Rev. D 92 (2015) 075002 [arXiv: 1503.05491] [INSPIRE].

[68] R. Foot, H. Lew, X.G. He and G.C. Joshi, Seesaw neutrino masses induced by a triplet of leptons, Z. Phys. C 44 (1989) 441 [INSPIRE]. 
[69] R. Franceschini, T. Hambye and A. Strumia, Type-III see-saw at LHC, Phys. Rev. D 78 (2008) 033002 [arXiv:0805.1613] [INSPIRE].

[70] Y. Cai, T. Han, T. Li and R. Ruiz, Lepton number violation: seesaw models and their collider tests, Front. Phys. 6 (2018) 40 [arXiv:1711.02180].

[71] S.R. Elliott and J. Engel, Double beta decay, J. Phys. G 30 (2004) R183 [hep-ph/0405078] [INSPIRE].

[72] P. Benes, A. Faessler, F. Simkovic and S. Kovalenko, Sterile neutrinos in neutrinoless double beta decay, Phys. Rev. D 71 (2005) 077901 [hep-ph/0501295] [INSPIRE].

[73] W. Rodejohann, Neutrino-less double beta decay and particle physics, Int. J. Mod. Phys. E 20 (2011) 1833 [arXiv:1106.1334] [INSPIRE].

[74] OPAL collaboration, Limits on neutral heavy lepton production from Z0 decay, Phys. Lett. B 247 (1990) 448 [INSPIRE].

[75] L3 collaboration, Search for isosinglet neutral heavy leptons in Z0 decays, Phys. Lett. B 295 (1992) 371 [INSPIRE].

[76] DELPHI collaboration, Search for neutral heavy leptons produced in Z decays, Z. Phys. C 74 (1997) 57 [Erratum ibid. C $\mathbf{7 5}$ (1997) 580] [INSPIRE].

[77] F. del Aguila, E. Laermann and P.M. Zerwas, Exotic $E_{6}$ particles in $e^{+} e^{-}$annihilation, Nucl. Phys. B 297 (1988) 1 [INSPIRE].

[78] W. Buchmüller and C. Greub, Heavy Majorana neutrinos in electron-positron and electron-proton collisions, Nucl. Phys. B 363 (1991) 345 [InSPIRE].

[79] A. Djouadi, New fermions at $e^{+} e^{-}$colliders. 1. Production and decay, Z. Phys. C 63 (1994) 317 [hep-ph/9308339] [INSPIRE].

[80] G. Azuelos and A. Djouadi, New fermions at $e^{+} e^{-}$colliders. 2. Signals and backgrounds, Z. Phys. C 63 (1994) 327 [hep-ph/9308340] [INSPIRE].

[81] J. Gluza and M. Zralek, Is there a chance to find heavy neutrinos in future linear colliders?, Phys. Lett. B 372 (1996) 259 [hep-ph/9510407] [INSPIRE].

[82] J. Gluza, J. Maalampi, M. Raidal and M. Zralek, Heavy neutrino mixing and single production at linear collider, Phys. Lett. B 407 (1997) 45 [hep-ph/9703215] [INSPIRE].

[83] L3 collaboration, Search for heavy isosinglet neutrinos in $e^{+} e^{-}$annihilation at $130 \mathrm{GeV}$ less than $S^{(1 / 2)}$ less than $189 \mathrm{GeV}$, Phys. Lett. B 461 (1999) 397 [hep-ex/9909006] [INSPIRE].

[84] L3 collaboration, Search for heavy isosinglet neutrino in $e^{+} e^{-}$annihilation at LEP, Phys. Lett. B 517 (2001) 67 [hep-ex/0107014] [INSPIRE].

[85] ALEPH collaboration, Measurement of W-pair production in $e^{+} e^{-}$collisions at centre-of-mass energies from $183 \mathrm{GeV}$ to $209 \mathrm{GeV}$, Eur. Phys. J. C 38 (2004) 147 [InSPIRE].

[86] P.S.B. Dev, A. Pilaftsis and U.-k. Yang, New production mechanism for heavy neutrinos at the LHC, Phys. Rev. Lett. 112 (2014) 081801 [arXiv:1308.2209] [INSPIRE].

[87] D. Alva, T. Han and R. Ruiz, Heavy Majorana neutrinos from $W \gamma$ fusion at hadron colliders, JHEP 02 (2015) 072 [arXiv: 1411.7305] [INSPIRE].

[88] R. Alonso, M. Dhen, M.B. Gavela and T. Hambye, Muon conversion to electron in nuclei in type-I seesaw models, JHEP 01 (2013) 118 [arXiv:1209.2679] [INSPIRE]. 
[89] F.F. Deppisch, P.S. Bhupal Dev and A. Pilaftsis, Neutrinos and collider physics, New J. Phys. 17 (2015) 075019 [arXiv: 1502.06541] [INSPIRE].

[90] S. Antusch, E. Cazzato and O. Fischer, Sterile neutrino searches at future $e^{-} e^{+}$, pp and $e^{-} p$ colliders, Int. J. Mod. Phys. A 32 (2017) 1750078 [arXiv:1612.02728] [inSPIRE].

[91] G. Cvetič, A. Das and J. Zamora-Saá, Probing heavy neutrino oscillations in rare $W$ boson decays, arXiv: 1805.00070 [INSPIRE].

[92] F. del Aguila, J.A. Aguilar-Saavedra and R. Pittau, Neutrino physics at large colliders, J. Phys. Conf. Ser. 53 (2006) 506 [hep-ph/0606198] [INSPIRE]. 